\title{
From massive gravity to modified general relativity
}

\author{
Scharf, Günter
}

\begin{abstract}
Massive gravity which has been constructed from a cohomological formulation of gauge invariance by means of the descent equations is here investigated in the classical limit. Gauge invariance requires a vector-graviton field $\mathrm{v}$ coupled to the massive tensor field $\mathrm{h}$. In the limit of vanishing graviton mass the v-field does not decouple. On the classical level this leads to a modification of general relativity. The contribution of the v-field to the energy-momentum tensor can be interpreted as dark matter density and pressure. We solve the modified field equations in the simplest spherically symmetric geometry
\end{abstract}

DOI: https://doi.org/10.1007/s10714-009-0864-0

Posted at the Zurich Open Repository and Archive, University of Zurich

ZORA URL: https://doi.org/10.5167/uzh-156168

Journal Article

Published Version

Originally published at:

Scharf, Günter (2010). From massive gravity to modified general relativity. General Relativity and Gravitation, 42(3):471-487.

DOI: https://doi.org/10.1007/s10714-009-0864-0 


\title{
From massive gravity to modified general relativity
}

\author{
Günter Scharf
}

Received: 8 November 2008 / Accepted: 6 July 2009 / Published online: 24 July 2009

(C) Springer Science+Business Media, LLC 2009

\begin{abstract}
Massive gravity which has been constructed from a cohomological formulation of gauge invariance by means of the descent equations is here investigated in the classical limit. Gauge invariance requires a vector-graviton field $v$ coupled to the massive tensor field $h_{\mu \nu}$. In the limit of vanishing graviton mass the $v$-field does not decouple. On the classical level this leads to a modification of general relativity. The contribution of the $v$-field to the energy-momentum tensor can be interpreted as dark matter density and pressure. We solve the modified field equations in the simplest spherically symmetric geometry.
\end{abstract}

Keywords Quantum gravity · Gauge theory · Dark matter

\section{Introduction}

Massive gravity is a controversial subject because it is very difficult to construct it starting from a classical Lagrangian theory. The reason is that the Brout-EnglertHiggs mechanism meets severe problems in the case of the massive spin-2 graviton [1]. On the other hand, there exists a powerful method to find the quantum theory directly without using any classical Lagrangian. Instead we start from a gauge structure on the asymptotic free fields and derive the gauge couplings from a cohomological formulation of gauge invariance. This method works for massless and massive gauge theories equally well. For spin-1 theories this was demonstrated in the monograph [2], where the massless spin-2 case is also treated. The massive spin-2 theory was first investigated in [3]. The most elegant way to obtain the theory is by assuming the

G. Scharf $(\varangle)$

Institut für Theoretische Physik, Universität Zürich,

Winterthurerstr. 190, 8057 Zurich, Switzerland

e-mail: scharf@physik.unizh.ch 
gauge invariance condition for all chronological products in the form of the descent equations [4]. These give the total interaction Lagrangian including ghost couplings and the necessary coupling to a vector-graviton field. This vector-graviton field $v^{\lambda}$ is of fundamental importance because without it a massive spin-2 gauge theory is impossible. So the main purpose of this paper is to analyze the physical consequences of this new actor in the drama of gravity

When a spin-1 vector boson gets a mass a Higgs field is necessary in order to save gauge invariance in higher orders. Therefore one would expect some gravitational Higgs field also in massive gravity. However, the theory is gauge invariant in second order without any additional field [3], and in third order the anomalies checked so far also cancel. A gravitational Higgs seems not to exist.

There is a second option in massive gravity. A massive spin-2 particle has five degrees of freedom in contrast to 2 of the massless graviton. It turns out that there are even 6 physical modes in massive gravity [5]. In gauge theory there is always some freedom to choose the physical states. In order to have a smooth massless limit $m \rightarrow 0$ of the massive theory, one is forced to choose the 6 physical states as follows: two of them are the transversal modes of the massless graviton, the remaining four are generated by the vector-graviton field $v^{\lambda}$ mentioned already. Therefore, the $v$-field describes physical degrees of freedom. The crucial fact is that for $m \rightarrow 0$ the $v^{\lambda}$ does not decouple from the symmetric tensor field $h^{\mu \nu}$ which corresponds to the classical $g^{\mu v}$ of Einstein. Consequently, the massless limit of massive gravity is not general relativity; there is a modification due to the (now massless) vector graviton field $v^{\lambda}$. We will show that this modification gives rise to an additional force which looks as if it comes from a dark matter density.

The paper is organized as follows. In the next section, we introduce the asymptotic free quantum fields of the theory and the gauge structure. Using the lowest order coupling derived previously by gauge invariance $[3,4]$ we discuss the limit of vanishing graviton mass and then the classical limit. The surviving coupling term between the tensor- and vector-graviton field leads to modified general relativity. In Sect. 3, we discuss the corresponding modified Schwarzschild (vacuum) solution. In the last section we present a general method of integration of the new field equations.

\section{Massive quantum gravity and its classical limit}

Our starting point is the massive spin-2 quantum gauge theory on Minkowski space which we have called massive gravity for short. The basic free asymptotic fields of this theory are the symmetric tensor field $h^{\mu v}(x)$ with arbitrary trace, the fermionic ghost $u^{\mu}(x)$ and anti-ghost $\tilde{u}^{\mu}(x)$ fields and the vector-graviton field $v^{\lambda}(x)$. They all satisfy the Klein-Gordon equation with the same mass $m$

$$
\left(\square+m^{2}\right) h^{\mu v}=0=\left(\square+m^{2}\right) u^{\mu}=\left(\square+m^{2}\right) \tilde{u}^{\mu}=\left(\square+m^{2}\right) v^{\lambda} .
$$

The relation between $h^{\mu \nu}$ and Einstein's $g^{\mu \nu}$ will be discussed below (2.12). The fields must be quantized as follows $[3,4]$ 


$$
\begin{aligned}
{\left[h^{\alpha \beta}(x), h^{\mu v}(y)\right] } & =-\frac{i}{2}\left(\eta^{\alpha \mu} \eta^{\beta v}+\eta^{\alpha \nu} \eta^{\beta \mu}-\eta^{\alpha \beta} \eta^{\mu v}\right) D_{m}(x-y) \\
\left\{u^{\mu}(x), \tilde{u}^{v}(y)\right\} & =i \eta^{\mu v} D_{m}(x-y) \\
{\left[v^{\mu(x)}, v^{v}(y)\right] } & =\frac{i}{2} \eta^{\mu v} D_{m}(x-y)
\end{aligned}
$$

and zero otherwise. Here, $D_{m}(x)$ is the Jordan-Pauli distribution with mass $m$ and $\eta^{\mu \nu}=\operatorname{diag}(1,-1,-1,-1)$ the Minkowski tensor. There are no classical gauge constraints to be imposed; the latter hold on the physical states only [2].

The gauge structure on these fields is defined through a nilpotent gauge charge operator $Q$ satisfying

$$
Q^{2}=0, \quad Q \Omega=0
$$

where $\Omega$ is the Fock vacuum and

$$
\begin{aligned}
& d_{Q} h^{\mu v}=\left[Q, h^{\mu v}\right]=-\frac{i}{2}\left(\partial^{v} u^{\mu}+\partial^{\mu} u^{v}-\eta^{\mu v} \partial_{\alpha} u^{\alpha}\right) \\
& d_{Q} u^{\mu} \stackrel{\text { def }}{=}\{Q, u\}=0 \\
& d_{Q} \tilde{u}^{\mu} \stackrel{\text { def }}{=}\left\{Q, \tilde{u}^{\mu}\right\}=i\left(\partial_{\nu} h^{\mu v}-m v^{\mu}\right) \\
& d_{Q} v^{\mu} \stackrel{\text { def }}{=}\left[Q, v^{\mu}\right]=-\frac{i}{2} m u^{\mu} .
\end{aligned}
$$

The vector-graviton field $v^{\lambda}$ is necessary for nilpotency of $Q$. In fact, in order to get $d_{Q}^{2} \tilde{u}=0$ from (2.5), the additional term $-m v^{\mu}$ is indispensable.

The coupling $T(x)$ between these fields follows from the gauge invariance condition [2]

$$
d_{Q} T(x)=i \partial_{\alpha} T^{\alpha}(x)
$$

where $T$ and $T^{\alpha}$ are normally ordered polynomials with ghost number 0 and 1 , respectively. In addition we may require the descent equations [4]

$$
\begin{gathered}
\partial_{Q} T^{\alpha}=\left[Q, T^{\alpha}\right]=i \partial_{\beta} T^{\alpha \beta} \\
{\left[Q, T^{\alpha \beta}\right]=i \partial_{\gamma} T^{\alpha \beta \gamma}}
\end{gathered}
$$

where the new $T$ 's are antisymmetric in the Lorentz indices. The essentially unique coupling derived from (2.7-9) is given by $[3,4]$

$$
\begin{aligned}
T= & h^{\alpha \beta} \partial_{\alpha} h \partial_{\beta} h-2 h^{\alpha \beta} \partial_{\alpha} h^{\mu \nu} \partial_{\beta} h_{\mu \nu}-4 h_{\alpha \beta} \partial_{\nu} h^{\beta \mu} \partial_{\mu} h^{\alpha \nu}-2 h^{\alpha \beta} \partial_{\mu} h_{\alpha \beta} \partial^{\mu} h \\
& +4 h_{\alpha \beta} \partial^{v} h^{\alpha \mu} \partial_{\nu} h_{\mu}^{\beta}+4 h^{\alpha \beta} \partial_{\alpha} v_{\lambda} \partial_{\beta} v^{\lambda}+4 u^{\mu} \partial_{\beta} \tilde{u}_{\nu} \partial_{\mu} h^{\nu \beta}-4 \partial_{\nu} u_{\beta} \partial_{\mu} \tilde{u}^{\beta} h^{\mu \nu} \\
& +4 \partial_{\nu} u^{\nu} \partial_{\mu} \tilde{u}_{\beta} h^{\beta \mu}-4 \partial_{\nu} u^{\mu} \partial_{\mu} \tilde{u}_{\beta} h^{\nu \beta}-4 m u^{\alpha} \tilde{u}^{\beta} \partial_{\alpha} v_{\beta} \\
& -m^{2}\left(\frac{4}{3} h_{\mu \nu} h^{\mu \beta} h_{\beta}^{\nu}-h^{\mu \beta} h_{\mu \beta} h+\frac{1}{6} h^{3}\right) .
\end{aligned}
$$


Here $h=h_{\mu}^{\mu}$ is the trace and a coupling constant is arbitrary. The quartic couplings follow from second order gauge invariance and so on.

We consider the limit $m \rightarrow 0$ in the following. The massless limit of massive gravity is certainly a possible alternative to general relativity. The new physics comes from the surviving coupling term of the vector-graviton

$$
T_{v}=h^{\alpha \beta} \partial_{\alpha} v_{\lambda} \partial_{\beta} v^{\lambda}
$$

Since all fields are now massless we may compare $T_{v}$ with the photon-graviton coupling which in linear gravity is given by $h^{\alpha \beta} t_{\alpha \beta}$, where $t_{\alpha \beta}$ is the energy-momentum tensor of the electromagnetic field. It is traceless and conserved, $\partial^{\alpha} t_{\alpha \beta}=0$. Expressing the field tensor $F^{\mu \nu}$ by the vector potential $A^{\lambda}$ we get a coupling term of the form (2.11) plus four different additional terms. That means $T_{v}$ is different from photongraviton coupling, and in fact it does not come from a traceless conserved tensor $t_{\alpha \beta}$. For relativists this may be odd, but we must emphasize that our first principle is gauge invariance, general relativity is secondary.

To understand the situation better we consider a general coupling between $h^{\alpha \beta}$ and a symmetric tensor $t^{\mu \nu}$ with zero gauge variation $d_{Q} t^{\mu \nu}=0$ :

$$
T=h^{\alpha \beta}\left[a\left(\eta_{\alpha \mu} \eta_{\beta \nu}+\eta_{\alpha \nu} \eta_{\beta \mu}\right)+b \eta_{\alpha \beta} \eta_{\mu \nu}\right] t^{\mu \nu} .
$$

From (2.4) we get

$$
d_{Q} T=-\frac{i}{2}\left(\partial^{\alpha} u^{\beta}+\partial^{\beta} u^{\alpha}-\eta^{\alpha \beta} \partial_{\lambda} u^{\lambda}\right)\left(2 a t_{\alpha \beta}+b \eta_{\alpha \beta} t_{\mu}^{\mu}\right)
$$

which by partial integration is equal to

$$
=\operatorname{div}+i a u^{\beta} \partial^{\alpha} t_{\alpha \beta}-i(a+b) u^{\beta} \partial_{\beta} t_{\mu}^{\mu}
$$

where div is a divergence $i \partial_{\alpha} T^{\alpha}$. Now gauge invariance (2.7) requires the vanishing of the additional terms

$$
\partial^{\alpha} t_{\alpha \beta}=\left(1+\frac{b}{a}\right) \partial_{\beta} t_{\mu}^{\mu}
$$

Using $\partial^{2} v_{\lambda}=0$ we see that this condition is satisfied by $t_{\alpha \beta}=\partial_{\alpha} v_{\lambda} \partial_{\beta} v^{\lambda}$ (2.11) with $a=2, b=-1$ as it must be, but $t_{\alpha \beta}$ is neither conserved nor traceless.

To be able to do non-perturbative calculations in massive gravity we look for the classical theory corresponding to the coupling (2.10). It was shown in [2] Sect. 5.5 that the pure graviton couplings $h \partial h \partial h$ correspond to the Einstein-Hilbert Lagrangian

$$
L_{\mathrm{EH}}=\frac{2}{\kappa^{2}} \sqrt{-g} R
$$


in the following sense. We write the metric tensor as

$$
\sqrt{-g} g^{\mu \nu}=\eta^{\mu \nu}+\kappa h^{\mu \nu}
$$

and expand $L_{\mathrm{EH}}$ in powers of $\kappa$. Then the quadratic terms $O\left(\kappa^{0}\right)$ give the free theory, the cubic terms $O\left(\kappa^{1}\right)$ agree with the pure graviton coupling terms in (2.10) up to a factor 4 and and quartic and higher couplings follow from higher order gauge invariance, so that the full non-linear structure of general relativity is recovered.

To obtain $T_{v}$ (2.11) we must add

$$
L_{v}=\sqrt{-g} g^{\alpha \beta} \partial_{\alpha} v_{\lambda} \partial_{\beta} v^{\lambda}
$$

to $L_{\mathrm{EH}}$. One may be tempted to write covariant derivatives $\nabla_{\alpha}$ instead of partial derivatives in order to get a true scalar under general coordinate transformations. But this would produce quartic couplings containing $v_{\lambda}$ and such terms are absent in the quantum theory [3, Eq. (4.12)]. For the same reason the Lorentz index $\lambda$ in $v_{\lambda}$ is raised and lowered with the Minkowski tensor $\eta_{\mu \nu}$, but all other indices with $g_{\alpha \beta}$. Both together means that the vector graviton field $v_{\lambda}$ should be considered as four scalar fields in the classical theory. Then (2.13) is a scalar under general coordinate transformations as it must be. It is interesting to note that t'Hooft in his attempt to construct massive gravity [1] also needs the coupling to the four scalar fields. On the other hand, in the quantum gauge theory $v_{\lambda}$ is a genuine 4-vector field. To avoid misunderstanding we shall substitute the Greek summation index $\lambda$ by the Latin $n$ when we consider the classical theory in the following. The seemingly drastic change in character of the $v$-field has the following deeper reason. The quantum theory is a gauge theory on Minkowski space, consequently $v_{\lambda}$ is a Lorentz 4-vector here. The classical theory on the other hand is background independent so that the transformation properties of the 4 components $v_{n}$ are open. The matching of the two theories by expansion around flat space dictates the coupling and the properties of the $v_{n}$.

The total classical Lagrangian we have to study is now given by

$$
L_{\text {tot }}=L_{g}+L_{M}+L_{v}
$$

where

$$
L_{g}=\frac{c^{3}}{16 \pi G} \sqrt{-g} R,
$$

we have introduced the velocity of light and Newton's constant for later discussions. $L_{M}$ is given by the energy-momentum tensor $t_{\mu \nu}$ of ordinary matter as usual. Strictly speaking we should also add a term for the ghost coupling. At present we leave this out because probably these unphysical degrees of freedom don't contribute in the classical theory.

Calculating first the variational derivative with respect to $g_{\mu \nu}$ we get the following modified Einstein equations 


$$
R_{\mu \nu}-\frac{1}{2} g_{\mu \nu} R-\frac{8 \pi G}{c^{4}} t_{\mu \nu}=\frac{16 \pi G}{c^{3}}\left(-\partial_{\mu} v_{n} \partial_{\nu} v^{n}+\frac{1}{2} g_{\mu \nu} g^{\alpha \beta} \partial_{\alpha} v_{n} \partial_{\beta} v^{n}\right)
$$

Secondly the variation with respect to $v_{n}$ gives

$$
\partial_{\alpha}\left(\sqrt{-g} g^{\alpha \beta} \partial_{\beta} v^{n}\right)=0
$$

After multiplication with $1 / \sqrt{-g}$ this is the Laplace-Beltrami or rather the wave equation in the metric $g^{\alpha \beta}$. The coupled system (2.15), (2.16) is the modification of general relativity which we are going to study.

\section{Modified Schwarzschild solution}

As a first step in this study we want to construct a static spherically symmetric solution of the modified field equations. Following the convention of [6] we write the metric as

$$
d s^{2}=e^{v} c^{2} d t^{2}-r^{2}\left(d \vartheta^{2}+\sin ^{2} \vartheta \partial \varphi^{2}\right)-e^{\lambda} d r^{2}
$$

where $v(r)$ and $\lambda(r)$ are functions of $r$ only. It is straightforward to compute the Christoffel symbols and the Einstein tensor

$$
G_{\mu}^{\nu} \stackrel{\text { def }}{=} R_{\mu}^{\nu}-\frac{1}{2} \delta_{\mu}^{v} R
$$

The details are given in the appendix, the result is (5.3)

$$
\begin{aligned}
G_{0}^{0} & =e^{-\lambda}\left(\frac{\lambda^{\prime}}{r}-\frac{1}{r^{2}}\right)+\frac{1}{r^{2}} \\
G_{1}^{1} & =-e^{-\lambda}\left(\frac{\nu^{\prime}}{r}+\frac{1}{r^{2}}\right)+\frac{1}{r^{2}} \\
G_{2}^{2} & =G_{3}^{3}=-e^{-\lambda}\left(\frac{\nu^{\prime \prime}}{2}+\frac{v^{\prime 2}}{4}-\frac{\nu^{\prime} \lambda^{\prime}}{4}-\frac{\lambda^{\prime}}{2 r}+\frac{v^{\prime}}{2 r}\right) .
\end{aligned}
$$

In order to find the physical interpretation of the new terms in (2.15) we first consider the spherically symmetric problem assuming an energy momentum tensor of ordinary matter in the diagonal form

$$
t_{\alpha}^{\beta}=\operatorname{diag}\left(q c^{2},-p_{1},-p_{2},-p_{3}\right)
$$

where $q(r)$ is the (inertial) mass density and $p_{j}(r)$ are the pressure components. 
We now turn to the new contributions involving the four scalar fields $v^{n}$. We must first solve (2.16). Since

$$
\begin{aligned}
\partial_{\alpha}\left(\sqrt{-g} g^{\alpha \beta} \partial_{\beta}\right)= & -\sin \vartheta \frac{\partial}{\partial r}\left(e^{(\nu-\lambda) / 2} r^{2} \frac{\partial}{\partial r}\right)-e^{(\nu+\lambda) / 2} \frac{\partial}{\partial \vartheta}\left(\sin \vartheta \frac{\partial}{\partial \vartheta}\right) \\
& -\frac{e^{(\nu+\lambda) / 2}}{\sin \vartheta} \frac{\partial^{2}}{\partial \varphi^{2}}
\end{aligned}
$$

the Laplace-Beltrami equation (2.16) can be written in the form

$$
\frac{\partial}{\partial r}\left(e^{(\nu-\lambda) / 2} r^{2} \frac{\partial}{\partial r}\right) v_{\kappa}=e^{(v+\lambda) / 2} L^{2} v_{n},
$$

where $L^{2}$ is the quantum mechanical angular momentum operator squared. Consequently the angular dependence of $v_{n}$ is given by spherical harmonics $Y_{l}^{m}(\vartheta, \varphi)$ :

$$
v_{n}(r, \vartheta, \varphi)=v_{n}(r) Y_{l}^{m}(\vartheta, \varphi)
$$

and the radial function $v_{n}(r)$ satisfies the following radial equation

$$
\frac{\partial}{\partial r}\left(e^{(\nu-\lambda) / 2} r^{2} \frac{\partial v_{n}(r)}{\partial r}\right)=l(l+1) e^{(\nu+\lambda) / 2} v_{n}(r) .
$$

We only consider a single spherical harmonic here, because for reasons which will become clear soon we restrict ourselves to the case $l=0$. Then we get the simple result

$$
v_{n}^{\prime}(r)=\frac{C_{n}}{r^{2}} e^{(\lambda-v) / 2}
$$

here $C_{n}, n=0,1,2,3$ are constants of integration.

Now we are ready to write down the modified radial Einstein equations. In (2.15) we raise the second index

$$
\begin{gathered}
G_{\mu}^{\nu}=\frac{8 \pi G}{c^{3}}\left(\frac{1}{c} t_{\mu}^{\nu}+\delta_{\mu}^{\nu} g^{\alpha \beta} \partial_{\alpha} v_{n} \partial_{\beta} v^{n}-2 \partial_{\mu} v_{n} \partial^{\nu} v^{n}\right) . \\
G_{0}^{0}=e^{-\lambda}\left(\frac{\lambda^{\prime}}{r}-\frac{1}{r^{2}}\right)+\frac{1}{r^{2}}=\frac{8 \pi G}{c^{3}}\left(q(r) c+w_{0}(r)\right) \\
-G_{1}^{1}=e^{-\lambda}\left(\frac{\nu^{\prime}}{r}+\frac{1}{r^{2}}\right)-\frac{1}{r^{2}}=\frac{8 \pi G}{c^{3}}\left(\frac{p_{1}(r)}{c}+w_{1}(r)\right) \\
-G_{2}^{2}=e^{-\lambda}\left(\frac{\nu^{\prime \prime}}{2}+\frac{v^{\prime 2}}{4}-\frac{\nu^{\prime} \lambda^{\prime}}{4}-\frac{\lambda^{\prime}}{2 r}+\frac{v^{\prime}}{2 r}\right)=\frac{8 \pi G}{c^{3}}\left(\frac{p_{2}(r)}{c}+w_{2}(r)\right), \\
-G_{3}^{3}=e^{-\lambda}\left(\frac{\nu^{\prime \prime}}{2}+\frac{v^{\prime 2}}{4}-\frac{\nu^{\prime} \lambda^{\prime}}{4}-\frac{\lambda^{\prime}}{2 r}+\frac{v^{\prime}}{2 r}\right)=\frac{8 \pi G}{c^{3}}\left(\frac{p_{3}(r)}{c}+w_{3}(r)\right),
\end{gathered}
$$


where $w_{0}, w_{1}, w_{2}, w_{3}$ are the contributions from the $v$-field in (3.8). The two Eqs. (3.9), (3.10) together with (3.5) are sufficient to calculate all unknown functions $\lambda, v, v_{n}$, if $q(r)$ and $p_{j}(r)$ are known. That means the Eqs. (3.11), (3.12) are additional constraints. It is a delicate problem to satisfy them. If we assume $p_{2}=p_{3}$, we must have $w_{2}=w_{3}$ and this is only possible for $l=0$. Then $v_{n}$ depends on $r$ only. We restrict ourselves to this case in the following, i.e. $p_{j}=p$.

From (3.7) we now obtain the following contributions of the $v$-field

$$
\begin{aligned}
g^{11} \partial_{1} v_{n} \partial_{1} v^{n} & =\frac{1}{r^{4}} e^{-v}\left(C_{1}^{2}+C_{2}^{2}+C_{3}^{2}-C_{0}^{2}\right) \\
& \stackrel{\text { def }}{=} \frac{\rho_{0}}{r^{4}} e^{-v}
\end{aligned}
$$

and zero otherwise, so that

$$
w_{0}=\frac{\rho_{0}}{r^{4}} e^{-v}=w_{2}=w_{3}, \quad w_{1}=-w_{0}
$$

We see in (3.9) that the mass density $q(r)$ gets enlarged by the quantity $w_{0}(r) / c$ which we shall call dark density. We omit the notion "matter" because this energy density comes from the $v$-field, it is a relic of the massive graviton. Since the new contribution appears in (3.9) in exactly the same way as the ordinary mass density $q(r)$, it gravitates in the Newtonian limit as the normal matter. This is in agreement with what is assumed in dark matter simulations [7]. But there is also a modification in the pressure components (3.10-3.12) which remains to be investigated.

Next by suitable combination we simplify the equations. Adding (3.9) and (3.10) we get

$$
\frac{e^{-\lambda}}{r}\left(\lambda^{\prime}+v^{\prime}\right)=g\left(q+\frac{p}{c^{2}}\right)
$$

where

$$
g=\frac{8 \pi G}{c^{2}}
$$

Eliminating $p$ from (3.10) and (3.11) we obtain

$$
v^{\prime \prime}=2 e^{\lambda}\left(-\frac{1}{r^{2}}+2 g \frac{w_{0}}{c}\right)+\frac{v^{\prime} \lambda^{\prime}}{2}-\frac{v^{\prime 2}}{2}+\frac{\lambda^{\prime}+v^{\prime}}{r}+\frac{2}{r^{2}} .
$$

Next we differentiate (3.10) with respect to $r$ and use (3.17):

$$
g\left(\frac{p^{\prime}}{c^{2}}-\frac{w_{0}^{\prime}}{c}\right)=-e^{-\lambda} \frac{v^{\prime}}{2 r}\left(\lambda^{\prime}+v^{\prime}\right)+4 \frac{g}{c r} w_{0}
$$


Substituting (3.15) inhere we finally arrive at

$$
\frac{p^{\prime}}{c^{2}}=-\frac{v^{\prime}}{2}\left(q+\frac{p}{c^{2}}+\frac{2}{c} w_{0}\right)
$$

where (3.14) has been used. This differential equation for the pressure will be used instead of the second order equation (3.11) in the following.

For a first orientation we want to solve the equations neglecting the ordinary matter, i.e. $q=0=p$. This vacuum solution corresponds to the (outer) Schwarzschild solution in ordinary general relativity. The approximation is justified for $r$ large compared to the Schwarzschild radius $r_{S}=2 G M / c^{2}$ where $M$ is the total mass (see below). Then we find from (3.15) $\lambda^{\prime}+v^{\prime}=0$ or

$$
v(r)=D_{1}-\lambda(r)
$$

where $D_{1}$ is a constant of integration. Using this is (3.14) we have

$$
w_{0}=\frac{D_{2}}{r^{4}} e^{\lambda}
$$

where $D_{2}$ is another constant. Multiplying (3.9) by $r^{2}$ and introducing

$$
u=r e^{-\lambda}
$$

we write the equation in the form

$$
u^{\prime}=1-8 \pi \frac{D_{3}}{r y}
$$

where

$$
D_{3}=\frac{G D_{2}}{c^{3}}
$$

Although this Eq. (3.23) looks rather simple, we did not succeed in expressing the solution by known special functions, but a numerical solution can be easily obtained. The dark density $w_{0}$ (3.21) is given in terms of $u$ (3.22) by

$$
w_{0}=\frac{D_{2}}{r^{3} u(r)}
$$

For large $r$ we find the following power series expansion

$$
u=r-a_{1}+\frac{a_{2}}{r}+\frac{a_{3}}{r^{2}}+\cdots
$$


where

$$
a_{2}=8 \pi D_{3}, \quad a_{3}=4 \pi D_{3} a_{1}, \ldots
$$

$a_{1}$ is related to the total mass $M$ by the Schwarzschild relation

$$
a_{1}=\frac{2 G M}{c^{2}}=r_{S}
$$

The first two terms in (3.26) give the ordinary Schwarzschild solution, so that the modified solution approaches it for large $r$. The four terms (3.26) can be taken as the start of the numerical integration of (3.23) from large to small $r$. Note that the Schwarzschild horizon disappears if $D_{3}$ is not too small, but there remains a (naked) singularity at $r=0$. It is of the form

$$
u=4 \sqrt{-\pi D_{3} \log r+\alpha+\frac{\beta}{\log r}} .
$$

This is too weak to render $r^{2} w_{0}$ (3.25) integrable at $r=0$. We must conclude that this vacuum solution cannot be used for small $r$ where certainly matter has to be taken into account. For $D_{3}$ small enough there is a horizon defined by $u(r)=0$ as in the ordinary Schwarzschild solution.

Without better options we cut off the singularity by assuming $w_{0}=$ const. inside a core radius $r_{c}$, for $r \geq r_{c} w_{0}$ is given by (3.25). The core radius $r_{c}$ is determined as follows: We compute the total mass

$$
\begin{aligned}
M & =\frac{4 \pi}{c}\left(\int_{0}^{r_{c}}+\int_{r_{c}}^{\infty}\right) r^{2} w_{0}(r) \\
& =\frac{4 \pi}{c}\left(\frac{r_{c}^{3}}{3} w_{0}\left(r_{c}\right)+D_{2} \int_{r_{c}}^{\infty} \frac{d r}{r u}\right) \\
& =\frac{4 \pi}{c}\left(\frac{D_{2}}{3 u\left(r_{c}\right)}+\frac{D_{2}}{8 \pi D_{3}} \int_{r_{c}}^{\infty}\left(1-u^{\prime}\right) d r\right) \\
& =\frac{4 \pi}{3 c} \frac{D_{2}}{u\left(r_{c}\right)}-\frac{c^{2}}{2 G}\left(-a_{1}+r_{c}-u\left(r_{c}\right)\right)
\end{aligned}
$$

where (3.24) has been used. By (3.28) $M$ drops out and we get $r_{c}$ as the zero of the equation

$$
r_{c}-u\left(r_{c}\right)=\frac{8 \pi}{3} \frac{D_{3}}{u\left(r_{c}\right)} .
$$


Varying $D_{3}$ this enables us to vary $r_{c}$ and the length scale of the dark halo, if we keep its total mass $M$, i.e. $a_{1}$ (3.28) fixed. Indeed, the differential equation (3.23) is invariant under the scale transformation

$$
r \rightarrow \alpha r, \quad u \rightarrow \alpha u, \quad D_{3} \rightarrow \alpha^{2} D_{3}, \quad a_{1} \rightarrow \alpha a_{1}
$$

This implies that the length scale of the halo can be freely adjusted.

In astrophysical applications we measure $r$ in kpc. Let us consider a halo of $10^{14}$ solar masses, then $a_{1}(3.28)$ is equal to $10^{-2}$ (in kpc). Taking $D_{3}$ of the order $10^{-3}$ we find rather small core radii of a few kpc. Precisely, our numerical results agree with the linear relation

$$
r_{c}=3352 D_{3}
$$

Outside $r_{c}, u$ is given by the four terms in (3.26) up to promille accuracy. The corrections due to the pressure according to (3.19) are of the same magnitude. That means the tail of the dark density profile (3.25) can be approximated by

$$
w_{0}=\frac{D_{2}}{r} \frac{1}{r^{3}-a_{1} r^{2}+a_{2} r+a_{3}}, \quad r \geq r_{c} .
$$

On the other hand Navarro, Frenk and White [7] have found the following profile

$$
\varrho(r)=\frac{\varrho_{s}}{r\left(1+\frac{r}{r_{s}}\right)^{2}}
$$

from N-body simulations, where $r_{s}$ is the so-called scaling radius and $\varrho_{s}$ the scaling density. Obviously, this profile may describe the intermediate and inner part of the halo where visible matter cannot be neglected [8]. To make contact with this result we must calculate a solution with ordinary matter. Our result (3.30) gives the outer tail of the dark profile which, unfortunately, cannot yet be investigated by observations [8]. If no decrease of the rotation curves of galaxies is observed for large $r$ in the future, then we must consider solutions with non-flat asymptotics.

\section{Solution by quadratures}

To construct solutions with non-vanishing visible matter and isotropic pressure we proceed as follows. We eliminate $p(r)=p_{j}(r)$ in the two Eqs. (3.10) and (3.11):

$$
-\lambda^{\prime} e^{-\lambda}\left(\frac{v^{\prime}}{4}+\frac{1}{2 r}\right)=e^{-\lambda}\left(-\frac{v^{\prime \prime}}{2}-\frac{v^{\prime 2}}{4}+\frac{v^{\prime}}{2 r}+\frac{1}{r^{2}}\right)-\frac{1}{r^{2}}+2 g w_{0} .
$$

Introducing now

$$
y=e^{-\lambda}
$$


we have obtained a linear first order differential equation for $y(r)$ :

$$
f_{1}(r) y^{\prime}(r)=f_{2}(r) y+f_{3}(r)
$$

where

$$
\begin{aligned}
& f_{1}=\frac{v^{\prime}}{4}+\frac{1}{2 r} \\
& f_{2}=-\frac{v^{\prime \prime}}{2}-\frac{v^{\prime 2}}{4}+\frac{v^{\prime}}{2 r}+\frac{1}{r^{2}} \\
& f_{3}=-\frac{1}{r^{2}}+2 g w_{0} .
\end{aligned}
$$

Considering the dark density $w_{0}(r)(3.14)$ as given we can compute

$$
v(r)=\log \varrho_{0}-\log w_{0}-4 \log r .
$$

This enables us to express the coefficients $f_{1}, f_{2}, f_{3}$ in terms of $w_{0}$ :

$$
\begin{aligned}
& f_{1}=-\frac{w_{0}^{\prime}}{4 w_{0}}-\frac{1}{2 r} \\
& f_{2}=\frac{w_{0}^{\prime \prime}}{2 w_{0}}-\frac{3}{4} \frac{w_{0}^{\prime 2}}{w_{0}^{2}}-\frac{5}{2} \frac{w_{0}^{\prime}}{r w_{0}}-\frac{7}{r^{2}} \\
& f_{3}=-\frac{1}{r^{2}}+2 g w_{0}
\end{aligned}
$$

The remaining equation (3.9) then determines the mass density $q(r)$ of the normal matter.

The linear equation (4.3) can be simply solved by quadratures, so we have full control of the solution. The general solution $y(r)$ is the sum of a particular solution $y_{1}(r)$ of the inhomogeneous equation (4.3) plus a solution $y_{0}(r)$ of the homogeneous equation. We will soon realize that the solution is uniquely fixed by requiring that it approaches flat space for $r \rightarrow \infty$. To see this we set up an expansion of the form

$$
y=1-\frac{a_{1}}{r}+\frac{a_{2}}{r^{2}}+\cdots
$$

in agreement with (3.26). The dark density (3.14) must start as follows

$$
w_{0}=\frac{\varrho_{0}}{r^{4}}+\frac{d_{1}}{r^{5}}+\frac{d_{2}}{r^{6}}+\cdots
$$

A $1 / r^{3}$ tail is in contradiction with the flat space asymptotic $y \rightarrow 1$ for $r \rightarrow \infty$. From (4.12) and (4.8) we find

$$
f_{1}=\frac{1}{2 r}+\frac{d_{1}}{4 \varrho_{0}} \frac{1}{r^{2}}+\left(\frac{d_{2}}{2 \varrho_{0}}-\frac{d_{1}^{2}}{4 \varrho_{0}^{2}}\right) \frac{1}{r^{3}}+\cdots
$$


and similarly

$$
\begin{aligned}
& f_{2}=\frac{1}{r^{2}}+\frac{3 d_{1}}{2 \varrho_{0}} \frac{1}{r^{3}}+\left(\frac{4 d_{2}}{\varrho_{0}}-\frac{9 d_{1}^{2}}{4 \varrho_{0}^{2}}\right) \frac{1}{r^{4}}+\cdots \\
& f_{3}=-\frac{1}{r^{2}}+2 g\left(\frac{\varrho_{0}}{r^{4}}+\frac{d_{1}}{r^{5}}+\frac{d_{2}}{r^{6}}+\cdots\right) .
\end{aligned}
$$

Substituting all this into (4.3) we can determine the coefficients in (4.11). The result is

$$
y=1-\frac{d_{1}}{\varrho_{0} r}+\left(\frac{2 d_{1}^{2}}{\varrho_{0}^{2}}-\frac{2 d_{2}}{\varrho_{0}}-g \varrho_{0}\right) \frac{1}{r^{2}}+\cdots
$$

To carry out all integrations in terms of elementary functions we choose the following simple dark density profile

$$
w_{0}=\frac{w_{1}}{1+\left(r / r_{s}\right)^{4}}
$$

It contains two parameters $w_{1}$ and $r_{s}$ as most phenomenological dark matter profiles in the literature and has the correct asymptotic behavior. First we want to calculate the solution $y_{0}(r)$ of the homogeneous equation

$$
f_{1} y_{0}^{\prime}=f_{2} y_{0}
$$

which is given by

$$
y_{0}(r)=\exp \int^{r} \frac{f_{2}}{f_{1}} d x
$$

Now from (4.8) and (4.17) we get

$$
f_{1}=\frac{r^{4}-r_{s}^{4}}{2 r\left(r^{4}+r_{s}^{4}\right)} .
$$

In view of the integral in (4.19) it is convenient to use the original definitions (4.4), (4.5) which yield

$$
\frac{f_{2}}{f_{1}}=-2 \frac{f_{1}^{\prime}}{f_{1}}-v^{\prime}+\frac{4}{r}-\frac{2}{r^{2} f_{1}} .
$$

In our special case (4.17) this is equal to

$$
\frac{f_{2}}{f_{1}}=-2 \frac{f_{1}^{\prime}}{f_{1}}-v^{\prime}-\frac{8 r_{s}^{4}}{r\left(r^{4}-r_{s}^{4}\right)} .
$$


This can be easily integrated and exponentiated using (3.14)

$$
e^{-v}=r^{4} \frac{w_{0}}{\varrho_{0}}
$$

In this way we find the following solution (4.19)

$$
y_{0}(r)=\text { const. } \frac{r^{14}\left(r^{4}+r_{s}^{4}\right)}{\left(r^{4}-r_{s}^{4}\right)^{4}} .
$$

The solution of the linear homogeneous equation contains a free prefactor, of course. For $r \rightarrow \infty y_{0}(r)$ increases as $r^{2}$ which is in conflict with the asymptotic flatness $y \sim$ const. Consequently, the prefactor of $y_{0}$ has to be zero so that we get a unique solution of our problem as in the expansion (4.16).

From $y_{0}(r)$ on finds the solution $y_{1}(r)$ of the inhomogeneous equation by the wellknown method of variation of the constants. One makes the ansatz

$$
y_{1}(r)=a(r) y_{0}(r)
$$

where $y_{0}$ is given by (4.23) with prefactor 1 . Substituting this into (4.3) one obtains $a(r)$ by another integration:

$$
a(r)=\int^{r} \frac{f_{3}(x)}{f_{1}(x) y_{0}(x)} d x,
$$

where a constant of integration, i.e. the lower limit of the integral is free. The integration of the rational function (3.25) is elementary. The final result is

$$
\begin{aligned}
a(r)= & \frac{1}{r^{2}}-\frac{r_{s}^{4}}{r^{6}}+\frac{3}{5} \frac{r_{s}^{8}}{r^{10}}-\frac{r_{s}^{12}}{14 r^{14}} \\
& +4 g w_{1}\left[-2 \log \left(1+\frac{r_{s}^{4}}{r^{4}}\right)+\frac{7}{4} \frac{r_{s}^{4}}{r^{4}}-\frac{1}{2} \frac{r_{s}^{8}}{r^{8}}+\frac{1}{12} \frac{r_{s}^{12}}{r^{12}}\right]
\end{aligned}
$$

Substituting this back into (4.24) we get the desired unique solution which we denote by $y(r)$ again:

$$
\begin{aligned}
y(r)= & \frac{r^{4}+r_{s}^{4}}{\left(r^{4}-r_{s}^{4}\right)^{4}}\left\{r^{12}-r_{s}^{4} r^{8}+\frac{3}{5} r_{s}^{8} r^{4}-\frac{1}{7} r_{s}^{12}\right. \\
& \left.+g w_{1}\left[-8 r^{14} \log \left(1+\frac{r_{s}^{4}}{r^{4}}\right)+7 r_{s}^{4} r^{10}-2 r_{s}^{8} r^{6}+\frac{1}{3} r_{s}^{12} r^{2}\right]\right\} .
\end{aligned}
$$

The solution $y(r)$ has the correct asymptotic behavior $y \rightarrow 1$ for $r \rightarrow \infty$. However, there is a singularity at $r=r_{s}$. We ask whether this singularity is hidden by a horizon. A horizon corresponds to $y=\exp (-\lambda)=0$. So we look for a zero of the curly bracket in (4.27). If we insert realistic numbers for the dark matter density $w_{1}$ as 
given in [8], we find that the second line in (4.27) is completely neglegible compared to the first one due to the smallness of $g$ (3.16). The polynomial

$$
r^{12}-r_{s}^{4} r^{8}+\frac{3}{5} r_{s}^{8} r^{4}-\frac{1}{7} r_{s}^{12}
$$

has only one real zero $r_{0}<r_{s}$. Consequently the singularity can be seen from the outside, that means it is naked. Finally the mass density $q(r)$ of normal matter is obtained from (2.7) according to

$$
c q(r)=\frac{1}{g}\left(-\frac{y^{\prime}}{r}-\frac{y}{r^{2}}+\frac{1}{r^{2}}\right)-w_{0} .
$$

This also becomes singular at $r=r_{s}$.

If we substitute realistic values for the galaxy M33 [8], we find a singular radius $r_{s}$ of about $9 \mathrm{kpc}$. This is unacceptable. A similar conclusion can be drawn from the analysis of the following 3-parameter profile

$$
w_{0}=\frac{w_{1}}{r^{2}\left(1-a_{1} r+a_{2} r^{2}\right)} .
$$

Then the solution of the homogeneous equation (4.18) is given by

$$
y_{0}(r)=\frac{r^{-2-16 a_{2} / a_{1}^{2}}\left(1-a_{1} r+a_{2} r^{2}\right)}{\left|2 a_{2} r-a_{1}\right|^{16 a_{2} / a_{1}^{2}}} \exp \left(-\frac{8}{a_{1} r}\right) .
$$

This produces a singularity at $r_{s}=a_{1} / 2 a_{2}$. In this case we get $r_{s}$ around $10 \mathrm{kpc}$.

The occurrence of a singularity seems to be a general feature of the simple equation (4.3). It is a consequence of the integrability of the problem which is due to the assumption of an isotropic pressure $p(r)$ in (3.4). On the other hand the dark contributions $\mp w_{0}(r)$ in (3.10) and (3.11) appear with different signs. Since the normal matter is probably following the dark in a certain way, the pressure components $p_{j}(r)$ in (3.10) and (3.11) should not be the same. Therefore, a physically realistic assumption is an anisotropic energy-momentum tensor (3.4). We shall investigate this more general situation elsewhere. Another important question is whether the vector graviton field gets additional couplings from higher order gauge invariance.

Acknowledgments Illuminating discussions with Dan Grigore, Klaus Fredenhagen, Andreas Aste and computational aid by Roland Bernet are gratefully acknowledged.

\section{Appendix A}

We take the coordinates $x^{0}=c t, x^{1}=r, x^{2}=\vartheta, x^{3}=\varphi$ such that from (3.1) we have

$$
\begin{gathered}
g_{00}=e^{\nu}, \quad g_{11}=-e^{\lambda} \\
g_{22}=-r^{2}, \quad g_{33}=-r^{2} \sin ^{2} \vartheta
\end{gathered}
$$


and zero otherwise. The components with upper indices are the inverse of this. The determinant comes out to be

$$
g=\operatorname{det} g_{\mu \nu}=-e^{\nu+\lambda} r^{4} \sin ^{2} \vartheta
$$

Next we compute the Christoffel symbols

$$
\begin{gathered}
\Gamma_{10}^{0}=\frac{1}{2} v^{\prime}, \quad \Gamma_{00}^{1}=\frac{1}{2} v^{\prime} e^{\nu-\lambda}, \quad \Gamma_{11}^{1}=\frac{1}{2} \lambda^{\prime}, \quad \Gamma_{22}^{1}=-r e^{-\lambda} \\
\Gamma_{33}^{1}=-r e^{-\lambda} \sin ^{2} \vartheta, \quad \Gamma_{12}^{2}=\frac{1}{r}, \quad \Gamma_{33}^{2}=-\sin \vartheta \cos \vartheta, \quad \Gamma_{13}^{3}=\frac{1}{r}, \quad \Gamma_{23}^{3}=\cot \vartheta
\end{gathered}
$$

and zero otherwise, the prime denotes the derivative with respect to $r$ always. From this the contracted curvature tensor is given by

$$
R_{\mu \nu}=\frac{\partial \Gamma_{\mu \nu}^{\alpha}}{\partial x^{\alpha}}-\frac{\partial \Gamma_{\mu \alpha}^{\alpha}}{\partial x^{\nu}}+\Gamma_{\mu \nu}^{\alpha} \Gamma_{\alpha \varrho}^{\varrho}-\Gamma_{\mu \lambda}^{\varrho} \Gamma_{\nu \varrho}^{\lambda} .
$$

We obtain

$$
\begin{aligned}
& R_{00}=\frac{1}{2} e^{\nu-\lambda}\left(v^{\prime \prime}+\frac{1}{2} v^{\prime 2}-\frac{1}{2} v^{\prime} \lambda^{\prime}+\frac{2}{r} v^{\prime}\right) \\
& R_{11}=-\frac{1}{2} v^{\prime \prime}+\frac{1}{4} \lambda^{\prime}\left(v^{\prime}+\frac{4}{r}\right)-\frac{1}{4} v^{\prime 2} \\
& R_{22}=e^{-\lambda}\left(\frac{r}{2} \lambda^{\prime}-\frac{r}{2} v^{\prime}-1\right)+1 \\
& R_{33}=\sin ^{2} \vartheta R_{22} .
\end{aligned}
$$

For the scalar curvature we then find

$$
R=e^{-\lambda}\left(v^{\prime \prime}+\frac{1}{2} v^{\prime 2}-\frac{1}{2} v^{\prime} \lambda^{\prime}+2 \frac{v^{\prime}}{r}-2 \frac{\lambda^{\prime}}{r}+\frac{2}{r^{2}}\right)-\frac{2}{r^{2}} .
$$

Now we are ready to calculate

$$
\begin{aligned}
& G_{\mu}^{\nu} \stackrel{\text { def }}{=} R_{\mu}^{\nu}-\frac{1}{2} \delta_{\mu}^{v} R, \\
& G_{0}^{0}=e^{-\lambda}\left(\frac{\lambda^{\prime}}{r}-\frac{1}{r^{2}}\right)+\frac{1}{r^{2}} \\
& G_{1}^{1}=-e^{-\lambda}\left(\frac{\nu^{\prime}}{r}+\frac{1}{r^{2}}\right)+\frac{1}{r^{2}} \\
& G_{2}^{2}=G_{3}^{3}=-e^{-\lambda}\left(\frac{\nu^{\prime \prime}}{2}+\frac{\nu^{\prime 2}}{4}-\frac{\nu^{\prime} \lambda^{\prime}}{4}-\frac{\lambda^{\prime}}{2 r}+\frac{v^{\prime}}{2 r}\right) .
\end{aligned}
$$




\section{References}

1. t'Hooft G.: Unitarity in the Brout-Englert-Higgs Mechanism for Gravity. hep-th/0708.3184 v4

2. Scharf, G.: Quantum Gauge Theories-a True Ghost Story. Wiley-Interscience, New York (2001)

3. Grigore, D.R., Scharf, G.: Gen. Relativ. Gravit. 37(6), 1075 (2005). hep-th/0404157

4. Grigore, D.R., Scharf, G.: Class. Quant. Gravit. 25, 225008 (2008). hep-th/0711.0869

5. Berchtold, J.B., Scharf, G.: Gen. Relativ. Gravit. 39(9), 1489 (2007). hep-th/0702181

6. Adler, R., Bazin, M., Schiffer, M.: Introduction to General Relativity. McGraw-Hill, New York (1965)

7. Navarro, J.F., Frenk, C.S., White, S.D.M.: Astrophys. J. 462, 563 (1996)

8. Corbelli, E.: Mon. Not. Roy. Astron. Soc. 342199 (2003). astro-ph/0302318 